FZJ-IKP-TH-2008-24

HISKP-TH-08/24

\title{
On-Shell Consistency of the Rarita-Schwinger Field Formulation
}

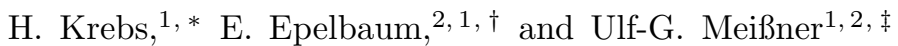 \\ ${ }^{1}$ Helmholtz-Institut für Strahlen- und Kernphysik (Theorie) and Bethe \\ Center for Theoretical Physics, Universität Bonn, D-53115 Bonn, Germany \\ ${ }^{2}$ Forschungszentrum Jülich, Institut für Kernphysik (IKP-3) and Jülich Center for Hadron Physics, \\ D-52425 Jülich, Germany
}

(Dated: June 20, 2013)

\begin{abstract}
We prove that any bilinear coupling of a massive spin-3/2 field can be brought into a gauge invariant form suggested by Pascalutsa by means of a non-linear field redefinition. The corresponding field transformation is given explicitly in a closed form and the implications for chiral effective field theory with explicit $\Delta(1232)$ isobar degrees of freedom are discussed.
\end{abstract}

PACS numbers: $13.75 . C s, 21.30 .-\mathrm{x}$

\section{INTRODUCTION}

A consistent formulation of a field theory involving interacting spin-3/2 degrees of freedom is an old and much studied problem, see e.g. Refs. [1, 2, 3]. Recently, it has attracted renewed interest in the context of chiral effective field theory of QCD with explicit $\Delta(1232)$ isobar degrees of freedom and its applications to e.g. pion-nucleon scattering or photo-nuclear processes, see Refs. 4, [5] for recent review articles.

In the commonly used Rarita-Schwinger formalism, the spin-3/2 field is represented by a vector-spinor $\psi_{\mu}$ (here and in what follows, we omit the spinor indices). It is well known how to write down the most general free Lagrangian for $\psi_{\mu}$ which describes the proper number of degrees of freedom [6]. The unphysical spin- $1 / 2$ degrees of freedom are projected out in the resulting free equations of motion. It is much more difficult to ensure the decoupling of the unphysical degrees of freedom in the case of interacting spin-3/2 fields. An elegant way to achieve this goal is to require that all interactions have the same type of gauge invariance as the kinetic term of the spin- $3 / 2$ field [7]. This requirement of gauge invariance is, however, not compatible with the non-linear realization of the chiral symmetry by Coleman, Callan, Wess and Zumino [8, 9], which is commonly adopted in chiral effective field theory and ensures the chiral invariance of the effective Lagrangian on a term-by-term basis. In this context, an important observation was made by Pascalutsa in Ref. [10], who showed that any gauge non-invariant linear coupling of the spin-3/2 fields can be brought into the gauge invariant form via a suitably chosen field redefinition. In that work, a conjecture was made that such a field redefinition should also be possible for bilinear couplings but no explicit form for this transformation was given. In this work we fill this gap and and prove that such a transformation indeed exists for arbitrary bilinear couplings. Moreover, we are able to give the transformation explicitly in a closed form.

Our paper is organized as follows. In section \we employ the functional integral technique to show that an interacting theory with gauge-invariant couplings of the spin-3/2 fields has the same constraints as the free theory. The non-linear field transformation which brings the gauge non-invariant bilinear couplings into the gauge invariant ones is discussed in section [II] A brief discussion on the so-called off-shell parameters can be found in section [V] In section $\nabla$ we show how our central result can be understood at the level of Feynman diagrams. We end with a summary and conclusions. Some technicalities are relegated to the appendix.

*Email: hkrebs@itkp.uni-bonn.de

${ }^{\dagger}$ Electronic address: e.epelbaum@fz-juelich.de

‡Electronic address: meissner@itkp.uni-bonn.de 


\section{SPIN-3/2 FIELDS WITH GAUGE-INVARIANT INTERACTIONS}

The most general Lagrangian for spin-3/2 fields can be written in the following form (after setting the pointtransformation parameter $A=-1)[\underline{6}, 16]$

$$
\mathcal{L}=\bar{\psi}^{\mu}\left((i \not)_{\mu \nu}-m_{\mu \nu}+V_{\mu \nu}\right) \psi^{\nu}=\bar{\psi}(i \not \partial-m+V) \psi .
$$

Here and in what follows, we use the short-hand notation:

$$
(i \not \partial)^{\mu \nu}=\gamma^{\mu \nu \alpha} i \partial_{\alpha}, \quad m^{\mu \nu}=\gamma^{\mu \nu} m,
$$

with $m$ being the mass of the spin-3/2 field and $\gamma^{\nu}$ the Dirac matrices. The quantities $\gamma^{\mu \nu}, \gamma^{\mu \nu \alpha}$ are defined according to

$$
\gamma^{\mu \nu}=\frac{1}{2}\left[\gamma^{\mu}, \gamma^{\nu}\right], \quad \gamma^{\mu \nu \alpha}=\frac{1}{2}\left\{\gamma^{\mu \nu}, \gamma^{\alpha}\right\}
$$

and are completely antisymmetric with respect to the Dirac indices. We will also need the inverse of the mass operator $m^{\mu \nu}$ which in $d$ dimensions takes the form

$$
\left[\frac{1}{m}\right]^{\mu \nu}=-\frac{1}{m}\left(g^{\mu \nu}+\frac{1}{1-d} \gamma^{\mu} \gamma^{\nu}\right)
$$

Notice that because of the completely antisymmetric nature of $\gamma^{\mu \nu \alpha}$, the free massless Lagrangian is invariant under the gauge transformation

$$
\psi_{\mu} \rightarrow \psi_{\mu}+\partial_{\mu} \epsilon
$$

where $\epsilon$ is an arbitrary spinor. We call the interacting field theory gauge-invariant if all interaction terms are invariant under this gauge transformation.

A gauge-invariant theory can be easily quantized using the standard path-integral technique. Indeed the path-integral formulation leads, by using the Faddeev-Popov trick, very naturally to the constraints which have the same form as in the free field theory. Let us denote the action of a gauge-invariant theory by

$$
S=S\left[\bar{\psi}_{\mu}, \psi_{\mu}, \phi\right]=S_{0}\left[\bar{\psi}_{\mu}, \psi_{\mu}, \phi\right]-\int d^{4} x \bar{\psi} m \psi
$$

Here we denote by $S_{0}$ the gauge invariant part of the theory

$$
S_{0}\left[\bar{\psi}_{\mu}+\partial_{\mu} \bar{\epsilon}, \psi_{\mu}+\partial_{\mu} \epsilon, \phi\right]=S_{0}\left[\bar{\psi}_{\mu}, \psi_{\mu}, \phi\right],
$$

and $\phi$ represents all other fields which interact with the spin-3/2 particle. The partition function in the path-integral formulation is given by

$$
Z=\int D \psi_{\mu} D \psi_{\mu}^{\dagger} D \phi \exp \{i S\}
$$

Inserting the Faddeev-Popov 1-operator

$$
1=\int D \epsilon D \bar{\epsilon} \delta\left(\left(\bar{\psi}_{\mu}+\partial_{\mu} \bar{\epsilon}\right) \gamma^{\mu}\right) \delta\left(\gamma_{\mu}\left(\psi^{\mu}-\partial^{\mu} \epsilon\right)\right) \frac{1}{\operatorname{Det}\left[-\gamma_{\mu} \partial^{\mu}\right]^{2}}
$$

we get

$$
Z=\int D \psi_{\mu} D \bar{\psi}_{\mu} D \phi D \epsilon D \bar{\epsilon} \delta\left(\left(\bar{\psi}_{\mu}+\partial_{\mu} \bar{\epsilon}\right) \gamma^{\mu}\right) \delta\left(\gamma_{\mu}\left(\psi^{\mu}-\partial^{\mu} \epsilon\right)\right) \frac{1}{\operatorname{Det}\left[-\gamma_{\mu} \partial^{\mu}\right]^{2}} \exp \{i S\}
$$

Using gauge-invariance we can rewrite the action as

$$
S\left[\bar{\psi}_{\mu}, \psi_{\mu}, \phi\right]=S\left[\bar{\psi}_{\mu}+\partial_{\mu} \bar{\epsilon}, \psi_{\mu}-\partial_{\mu} \epsilon, \phi\right]+\bar{\psi}_{\mu} \gamma^{\mu \nu} m \partial_{\nu} \epsilon+\bar{\epsilon} \partial_{\mu} \gamma^{\mu \nu} m \psi_{\nu}
$$


Substituting additionally the spin-3/2 fields by

$$
\bar{\psi}_{\mu} \rightarrow \bar{\psi}_{\mu}-\partial_{\mu} \bar{\epsilon}, \quad \psi_{\mu} \rightarrow \psi_{\mu}+\partial_{\mu} \epsilon,
$$

we obtain for the partition function

$$
Z=\frac{1}{\operatorname{Det}\left[-\gamma_{\mu} \partial^{\mu}\right]^{2}} \int D \psi_{\mu} D \bar{\psi}_{\mu} D \phi D \epsilon D \bar{\epsilon} \delta\left(\bar{\psi}_{\mu} \gamma^{\mu}\right) \delta\left(\gamma_{\mu} \psi^{\mu}\right) \exp \{i S\} \exp \left(-i m \int d^{4} x\left[\bar{\psi}_{\mu} \partial^{\mu} \epsilon+\bar{\epsilon} \partial^{\mu} \psi_{\mu}\right]\right)
$$

Finally, integrating over the spinor fields $\epsilon$ and $\bar{\epsilon}$ yields

$$
Z=\text { const } \int D \psi_{\mu} D \bar{\psi}_{\mu} D \phi \delta\left(\gamma_{\mu} \psi^{\mu}\right) \delta\left(\bar{\psi}_{\mu} \gamma^{\mu}\right) \delta\left(\partial_{\mu} \psi^{\mu}\right) \delta\left(\partial^{\mu} \bar{\psi}_{\mu}\right) \exp \{i S\}
$$

This is exactly the same expression as given in Eq. (46) of Ref. 7] which was derived for a specific gauge-invariant model using the Dirac-Faddeev quantization framework [11, 12, 13], see also Ref. 14] for a related work. Notice that the interacting theory has the same constraints as the free one. Clearly, this is the consequence of the fact that the gauge-invariance is only broken by the mass term in both theories, see [7] for an extended discussion.

\section{ON-SHELL EQUIVALENCE OF INTERACTIONS INVOLVING SPIN-3/2 FIELDS}

We now approach our main goal and demonstrate the on-shell equivalence of gauge-invariant and gauge non-invariant formulations of the interacting theories with the massive spin-3/2 fields extending the earlier work [10] to the case of bilinear couplings.

Consider the Lagrangian for interacting massive spin-3/2 particles given in its general form

$$
\mathcal{L}=\bar{\psi}(i \not \partial-m+V) \psi,
$$

where $V_{\mu \nu}$ represents an arbitrary local interaction. We claim that such a theory ${ }^{1}$ is on-shell equivalent to the gauge-invariant one described by the Lagrangian

$$
\mathcal{L}^{\prime}=\bar{\psi}\left(i \not \partial-m+i \not \partial \frac{1}{m} V \frac{1}{m}\left[1-(i \not \partial+m) \frac{1}{m} V \frac{1}{m}\right]^{-1} i \not \partial\right) \psi .
$$

To prove this statement we rely on the equivalence-theorem [8, 15] which states that two field theories are equivalent on mass shell provided there exists a field transformation

$$
\psi \rightarrow \psi+P[\psi, \phi]
$$

which transforms one Lagrangian into another. Here, $P[\psi, \phi]=\mathcal{O}\left(\psi^{2}, \psi \phi\right)$ denotes a local polynomial of the fields $\psi, \phi$ and their derivatives. In the following, we will show that the original Lagrangian in Eq. (3.1) can be brought into the gauge-invariant form given in Eq. (3.2) by the following field transformation:

$$
\psi \rightarrow S^{-1}[X-1]^{-1} S \psi \quad \text { and } \quad \bar{\psi} \rightarrow \bar{\psi} \bar{S}[\bar{X}-1]^{-1} \bar{S}^{-1},
$$

where the operators $X$ and $\bar{X}$ are defined via

$$
X=1+\left[1-\frac{1}{m} V \frac{1}{m}(i \not \partial+m)\right]^{1 / 2} \quad \text { and } \quad \bar{X}=1+\left[1-(i \not \partial+m) \frac{1}{m} V \frac{1}{m}\right]^{1 / 2} .
$$

Further, the quantities $S$ and $\bar{S}$ have the form

$$
S=\frac{1}{2}\left[X-\frac{1}{m} V\right]=\frac{1}{2} X\left[1-X^{-1} \frac{1}{m} V\right] \quad \text { and } \quad \bar{S}=\frac{1}{2}\left[\bar{X}-V \frac{1}{m}\right]=\frac{1}{2}\left[1-V \frac{1}{m} \bar{X}^{-1}\right] \bar{X} .
$$

\footnotetext{
1 Throughout this work we refer to the naive Feynman rules in the case of gauge non-invariant couplings.
} 
Notice that the field redefinition specified in Eq. (3.4) is clearly of the form of Eq. (3.3).

To prove the equivalence of the two Lagrangians we have to show that

$$
\bar{S}[\bar{X}-1]^{-1} \bar{S}^{-1}(i \not \partial-m+V) S^{-1}[X-1]^{-1} S \stackrel{!}{=} i \not \partial-m+i \not \partial \frac{1}{m} V \frac{1}{m}\left[1-(i \not \partial+m) \frac{1}{m} V \frac{1}{m}\right]^{-1} i \not \partial .
$$

Using Eq. (A.4) from Appendix A to rewrite the right-hand side of the above equation, so that the relation to be verified becomes

$$
\bar{S}^{-1}(i \not \partial-m+V) S^{-1} \stackrel{!}{=}[\bar{X}-1] \bar{S}^{-1}(i \not \partial-m+V) S^{-1}[X-1]+4(i \not \partial+m)-2[\bar{X}-1] \bar{S}^{-1}(i \not \partial+m)-2(i \not \partial+m) S^{-1}[X-1] .
$$

The left-hand side of this relation can be rewritten in the following way:

$$
\begin{aligned}
\bar{S}^{-1}(i \not \partial-m+V) S^{-1} & =\bar{S}^{-1}\left(2 \bar{S} \bar{X}^{-1}(i \not \partial+m)-2 m S\right) S^{-1} \\
& =2\left(\bar{X}^{-1}(i \not \partial+m) S^{-1}-\bar{S}^{-1} m\right) .
\end{aligned}
$$

In the first line we used Eq. (A.6) of Appendix A. The relation to prove then becomes

$$
4(i \not \partial+m)-2[\bar{X}-1] \bar{S}^{-1}(i \not \partial+m)-2 \bar{X}^{-1}(i \not \partial+m) S^{-1} X+2 \bar{S}^{-1} m X-2 \bar{X} \bar{S}^{-1} m[X-1] \stackrel{!}{=} 0
$$

Using the commutation-like relation

$$
m\left[1-X^{-1} \frac{1}{m} V\right]^{-1}=\left[1-V \frac{1}{m} \bar{X}^{-1}\right]^{-1}
$$

we can express $S^{-1}$ as follows

$$
S^{-1}=2\left[1-X^{-1} \frac{1}{m} V\right]^{-1} X^{-1}=2 \frac{1}{m}\left[1-V \frac{1}{m} \bar{X}^{-1}\right]_{m X^{-1}}^{-1}=\frac{1}{m} V \frac{1}{m} \bar{S}^{-1} m X^{-1}+2 X^{-1} .
$$

Let us denote the left-hand side of Eq. (3.10), which we want to show to be equal to zero, by $Y$. Inserting the above expression for $S^{-1}$ into the third term in Eq. (3.10) we obtain:

$$
Y=4(i \not \partial+m)-2[\bar{X}-1] \bar{S}^{-1}(i \not \partial+m)-2 \bar{X}^{-1}(i \not \partial+m) \frac{1}{m} V \frac{1}{m} \bar{S}^{-1} m-4 \bar{X}^{-1}(i \not \partial+m)+2 \bar{S}^{-1} m X-2 \bar{X} \bar{S}^{-1} m[X-1] .
$$

For a further simplification, we need yet another useful relation which reads

$$
\bar{X}^{-1}(i \not \partial+m) \frac{1}{m} V \frac{1}{m}=2-\bar{X},
$$

and can be easily verified by multiplying both sides with $\bar{X}$ and using the definition Eq. (3.5). We now apply this relation to rewrite the third term in Eq. (3.13) which leads to

$$
\begin{aligned}
Y & =4(i \not \partial+m)-2[\bar{X}-1] \bar{S}^{-1}(i \not \partial+m)-4 \bar{X}^{-1}(i \not \partial+m)-2[\bar{X}-1] \bar{S}^{-1} m[X-2] \\
& =4(i \not \partial+m)-2[\bar{X}-1] \bar{S}^{-1}(i \not \partial+m)-4 \bar{X}^{-1}(i \not \partial+m)+2[\bar{X}-1] \bar{S}^{-1} m X^{-1} \frac{1}{m} V \frac{1}{m}(i \not \partial+m) \\
& =4(i \not \partial+m)-2[\bar{X}-1] \bar{S}^{-1}(i \not \partial+m)-4 \bar{X}^{-1}(i \not \partial+m)+2[\bar{X}-1] \bar{S}^{-1} V \frac{1}{m} \bar{X}^{-1}(i \not \partial+m) .
\end{aligned}
$$

Here, in the second line we again used Eq. (3.14) to rewrite $X-2$. To end up with the third line we used the following commutation-like relation

$$
X^{-1} \frac{1}{m} V \frac{1}{m}=\frac{1}{m} V \frac{1}{m} \bar{X}^{-1} .
$$

Finally, putting together the second and the last term in the last line of Eq. (3.15) yields the desired result:

$$
\begin{aligned}
Y & =4(i \not \partial+m)-4[\bar{X}-1] \bar{S}^{-1} \bar{S} \bar{X}^{-1}(i \not \partial+m)-4 \bar{X}^{-1}(i \not \partial+m) \\
& =0 .
\end{aligned}
$$


Thus, the redefinition of the spin-3/2 field specified in Eq. (3.4) in the underlying gauge non-invariant Lagrangian $\mathcal{L}$ in Eq. (3.1) leads to the gauge-invariant form $\mathcal{L}^{\prime}$ given in Eq. (3.2). Notice that even if there is only a finite number of interaction terms in the original Lagrangian, the transformed Lagrangian always contains an infinite number of terms.

Let us now briefly discuss possible linear couplings like the couplings of the $\Delta$ to a nucleon fields. Once the bilinear terms are gauge-invariant the entire Lagrangian can be transformed into gauge-invariant form by much simpler transformation which was extensively discussed by Pascalutsa [10]. We briefly repeat the arguments. We start with the Lagrangian

$$
\mathcal{L}=\bar{\psi}(i \not \partial-m+V) \psi+\bar{\psi} \mathcal{O}_{\Delta N} N+\bar{N} \mathcal{O}_{N \Delta} \psi+\ldots,
$$

where $N$ and $\bar{N}$ represent nucleon fields, the operators $V, \mathcal{O}_{\Delta N}, \mathcal{O}_{N \Delta}$ represent interactions between nucleons and deltas and pions or other fields and the ellipsis represent all other possible interactions which do not include delta degrees of freedom. After the transformation Eq. (3.4) we obtain the Lagrangian

$$
\mathcal{L}^{\prime}=\bar{\psi}\left(V_{\Delta \Delta}-m\right) \psi+\bar{\psi} \tilde{V}_{\Delta N} N+\bar{N} \tilde{V}_{N \Delta} \psi+\ldots,
$$

where the operators $V_{\Delta \Delta}, \tilde{V}_{\Delta N}$ and $\tilde{V}_{N \Delta}$ are given as follows

$$
\begin{aligned}
V_{\Delta \Delta} & =i \not \partial+i \not \partial \frac{1}{m} V \frac{1}{m}\left[1-(i \not \partial+m) \frac{1}{m} V \frac{1}{m}\right]^{-1} i \not \partial, \\
\tilde{V}_{\Delta N} & =\bar{S}[\bar{X}-1]^{-1} \bar{S}^{-1} \mathcal{O}_{\Delta N}, \\
\tilde{V}_{N \Delta} & =\mathcal{O}_{N \Delta} S^{-1}[X-1]^{-1} S .
\end{aligned}
$$

Note that the interaction $V_{\Delta \Delta}$ includes only gauge-invariant vertices. However the interactions $\tilde{V}_{\Delta N}$ and $\tilde{V}_{N \Delta}$ are in general not gauge-invariant. In order to bring them into a gauge-invariant form let us shift the delta fields like

$$
\bar{\psi}_{\mu} \rightarrow \bar{\psi}_{\mu}+\bar{\xi}_{\mu}, \quad \psi_{\mu} \rightarrow \psi_{\mu}+\xi_{\mu}
$$

where $\bar{\xi}_{\mu}$ and $\xi_{\mu}$ are not yet specified but assumed to have the property not to depend on the delta fields. The transformed Lagrangian reads

$$
\mathcal{L}^{\prime \prime}=\bar{\psi}\left(V_{\Delta \Delta}-m\right) \psi+\bar{\xi} V_{\Delta \Delta} \psi+\bar{\psi} V_{\Delta \Delta} \xi+\bar{\psi}\left(\tilde{V}_{\Delta N} N-m \xi\right)+\left(\bar{N} \tilde{V}_{N \Delta}-\bar{\xi} m\right) \psi+\ldots .
$$

In order to get a gauge-invariant form we now fix the fields $\xi_{\mu}$ and $\bar{\xi}_{\mu}$ to

$$
\bar{\xi}=\bar{N} \tilde{V}_{N \Delta} \frac{1}{m}, \quad \xi=\frac{1}{m} \tilde{V}_{\Delta N} N .
$$

With this choice the Lagrangian becomes gauge-invariant:

$$
\mathcal{L}^{\prime \prime}=\bar{\psi}\left(V_{\Delta \Delta}-m\right) \psi+\bar{\psi} V_{\Delta N} N+\bar{N} V_{N \Delta} \psi+\ldots
$$

where the gauge-invariant delta-nucleon interactions are given by

$$
V_{\Delta N}=V_{\Delta \Delta} \frac{1}{m} \tilde{V}_{\Delta N}, \quad V_{N \Delta}=\tilde{V}_{N \Delta} \frac{1}{m} V_{\Delta \Delta} .
$$

The equivalence of the two formulations by means of the non-linear field redefinition as discussed above has important consequences for calculations within chiral effective field theory. It implies that S-matrix elements can be calculated from the standard effective Lagrangian with the chiral symmetry being realized on a term-by-term basis using naive Feynman rules.

\section{DEPENDENCE ON OFF-SHELL PARAMETERS}

Let us briefly discuss what happens with the off-shell parameters which account for the unphysical spin- $1 / 2$ degrees of freedom. The original interaction $\bar{\psi} V \psi$ can be written as a series of interactions with different off-shell parameters $Z_{i}$ and $Z_{i}^{\prime}$

$$
\bar{\psi} V \psi=\sum_{i} \bar{\psi} \Theta\left(Z_{i}^{\prime}\right) V_{i} \Theta\left(Z_{i}\right) \psi+\text { h.c., } \quad \Theta\left(Z_{i}\right)_{\mu \nu}=g_{\mu \nu}+Z_{i} \gamma_{\mu} \gamma_{\nu}
$$


Multiplication of the off-shell function $\Theta\left(Z_{i}\right)$ with $\frac{1}{m} i \not \partial \psi$ or $\bar{\psi} i \not \partial \frac{1}{m}$ from right or left, respectively, projects out the off-shell parameters $Z_{i}$ :

$$
\begin{aligned}
{\left[\Theta\left(Z_{i}\right) \frac{1}{m} i \not \partial\right]_{\mu} } & =\left[\frac{1}{m} i \not \partial \psi\right]_{\mu}+Z_{i} \gamma_{\mu} \gamma^{\lambda}\left[\frac{1}{m}\right]_{\lambda \nu}[i \not \partial]^{\nu} \\
& =\left[\frac{1}{m} i \not \partial \psi\right]_{\mu}+\frac{Z_{i}}{m} \frac{1}{d-1} \gamma_{\mu} \gamma_{\nu}[i \not \partial \psi]^{\nu} \\
& =\left[\frac{1}{m} i \not \partial \psi\right]_{\mu}+\frac{Z_{i}}{m} \frac{d-2}{d-1} \gamma_{\mu} \gamma^{\nu \lambda} i \partial_{\lambda} \psi_{\nu} \\
& =\left[\frac{1}{m} i \not \partial\right]_{\mu}+\frac{Z_{i}}{m} \frac{d-2}{d-1} i \gamma_{\mu}\left(\partial^{\nu}-\partial_{\lambda} \gamma^{\lambda} \gamma^{\nu}\right) \psi_{\nu} \\
& =\left[\frac{1}{m} i \not \partial \psi\right]_{\mu} .
\end{aligned}
$$

In the third step we used the relation

$$
\gamma_{\alpha} \gamma^{\alpha \nu \lambda}=(d-2) \gamma^{\nu \lambda}
$$

In the last step the field constraints

$$
\gamma^{\nu} \psi_{\nu}=\partial^{\nu} \psi_{\nu}=0
$$

have been used. Similarly one can show that

$$
\left[\bar{\psi} i \not \partial \frac{1}{m} \Theta\left(Z_{i}^{\prime}\right)\right]_{\mu}=\left[\bar{\psi} i \not \partial \frac{1}{m}\right]_{\mu}
$$

by using the field constraints

$$
\bar{\psi}^{\nu} \gamma_{\nu}=\bar{\psi}^{\nu} \partial_{\nu}=0
$$

Due to these relations all the off-shell parameter contributions on the most left/right hand side of the gauge-invariant interactions $V_{\Delta \Delta}, V_{\Delta N}$ and $V_{N \Delta}$ are projected out. However, all the other off-shell parameter contributions are not zero but can be accounted for by counterterms. So in order for a given field theory to be independent of the offshell parameters counterterms are needed to absorb all the off-shell dependence. The theory becomes automaticaly an effective field theory with infinitely many counterterms. In this picture, the off-shell parameters play the role of renormalization scale: the counterterms do explicitely depend on the off-shell parameters in a way that all the calculated observables remain independent on them.

In the case of the theory with the chiral Lagrangian it remains to be clarified whether the originally present counterterms are sufficient in order to absorb all the off-shell parameter contributions. Note that the gauge-invariant interactions $V_{\Delta \Delta}, V_{\Delta N}$ and $V_{N \Delta}$ involve not only covariant derivatives which appear in the chiral Lagrangian. For this reason, it is not obvious that the chiral-invariant counterterms in the original Lagrangian can completely compensate the off-shell dependence. For a related discussion we refer to the work of Ellis and Tang [16, 17]. Notice further that at least in the case of pion-nucleon scattering at leading one-loop order the off-shell parameters can be absorbed in low-energy constants accompanying certain local interaction operators. This was confirmed in the explicit calculation by Fettes and Meißner [18] based on the so-called small scale expansion [19].

\section{INTERPRETATION IN TERMS OF FEYNMAN DIAGRAMS}

The general result proved algebraically in the previous section can also be understood on the level of Feynman diagrams. The underlying mechanism is even more evident in that case. To be specific, consider the generic Feynman diagram shown in Fig. 1 on mass-shell. We now show that the manipulations discussed in the previous section simply 


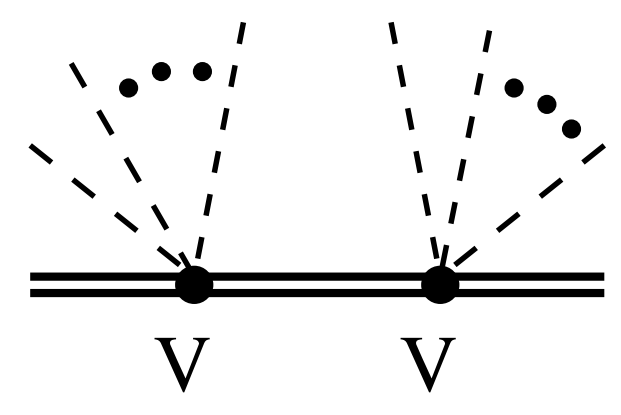

FIG. 1: Feynman diagram to illustrate the relation between gauge non-invariant and gauge-invariant formulations as described in the text. The solid double line represents the delta, while the dashed lines are arbitrary external fields like pions, photons and so on.

correspond to the partial fraction decomposition of the free Feynman propagator. The corresponding expression in operator form reads:

$$
i \not \partial \frac{1}{m} i V \frac{i}{i \not \partial-m} i V \frac{1}{m} i \not \partial=i \not \supset \frac{1}{m} i V \frac{1}{m} m \frac{i}{i \not \partial-m} m \frac{1}{m} i V \frac{1}{m} i \not \partial .
$$

Using the partial fraction decomposition

$$
m \frac{1}{i \not \partial-m} m=-(i \not \partial+m)+i \not \partial \frac{1}{i \not \partial-m} i \not \partial
$$

we can rewrite this diagram in a gauge-invariant form:

$$
i \not \partial \frac{1}{m} i V \frac{i}{i \not \partial-m} i V \frac{1}{m} i \not \partial=i \not \partial \frac{1}{m} i V \frac{1}{m} i \not \supset \frac{i}{i \not \partial-m} i \not \partial \frac{1}{m} i V \frac{1}{m} i \not \partial+i \not \supset \frac{1}{m} i V \frac{1}{m}(i \not \partial+m) \frac{1}{m} V \frac{1}{m} i \not \supset .
$$

Notice that because of the appearance of the operators $\frac{1}{m} i \not \supset$ and $i \not \partial \frac{1}{m}$ the first part includes only gauge invariant vertices which can not depend on off-shell parameters. The second term is just a vertex which accounts for all the offshell parameters. So by partial fraction decomposition one can naturally split the true spin- $3 / 2$ propagating structures which are gauge-invariant and all non-propagating spin- $1 / 2$ contributions which are collected into additional vertices.

\section{SUMMARY AND CONCLUSIONS}

In this paper, we have explicitely constructed a non-linear field transfromation that brings the interaction Lagrangian of spin-3/2 fields as given in Eq. (3.1) in a form that is invariant under gauge transformation Eq. (2.5). This proves a conjecture made in Ref. [10] where it was shown how a gauge non-invariant Lagrangian with linear couplings in the spin-3/2 fields can be brought into a gauge-invariant form via a suitably chosen field redefinition. We have also given a diagrammatic explanation of our main result, which allows to split the truly propagating spin-3/2 components from the non-propagating spin- $1 / 2$ contributions that are subsumed in local operators existing in the most general Lagrangian of deltas coupled to nucleons, pions and external fields.

\section{Acknowledgments}

We are grateful to Jambul Gegelia and Vladimir Pascalutsa for useful comments on the manuscript. The work of E.E. and H.K. was supported in parts by funds provided from the Helmholtz Association to the young investigator group "Few-Nucleon Systems in Chiral Effective Field Theory" (grant VH-NG-222) and through the virtual institute 
"Spin and strong QCD" (grant VH-VI-231). This work was further supported by the DFG (SFB/TR 16 "Subnuclear Structure of Matter") and by the EU Integrated Infrastructure Initiative Hadron Physics Project under contract number RII3-CT-2004-506078.

\section{APPENDIX A: SOME USEFUL OPERATOR RELATIONS}

In this appendix we list a number of helpful relations involving the operators $X, \bar{X}, S$ and $\bar{S}$, see Eqs. (3.5]3.6),

$$
\begin{gathered}
2 \bar{S}[\bar{X}-1]^{-1}-1=\left(1-V \frac{1}{m}\right)[\bar{X}-1]^{-1}, \quad[X-1]^{-1} 2 S-1=[X-1]^{-1}\left(1-\frac{1}{m} V\right) \\
{[\bar{X}-1]^{-2} m=[\bar{X}-1]^{-2}(i \not \partial+m) \frac{1}{m} V+m}
\end{gathered}
$$

as well as commutation-like relation

$$
(i \not \partial+m) X=\bar{X}(i \not \partial+m) .
$$

We now use the above equations to prove another important operator relation

$$
i \not \partial \frac{1}{m} V \frac{1}{m}[\bar{X}-1]^{-2} i \not \partial=\left[2 \bar{S}[\bar{X}-1]^{-1}-1\right](i \not \partial+m)\left[[X-1]^{-1} 2 S-1\right]-i \not \partial-m+V \text {, }
$$

used in section III to show the on-shell equivalence of the gauge-invariant and the gauge non-invariant bilinear interactions of the massive spin-3/2 field. A straightforward calculation yields:

$$
\begin{aligned}
i \not \partial \frac{1}{m} V \frac{1}{m}[\bar{X}-1]^{-2} i \not \partial & =(i \not \partial+m) \frac{1}{m} V \frac{1}{m}[\bar{X}-1]^{-2} i \not \partial-V \frac{1}{m}[\bar{X}-1]^{-2} i \not \partial \\
& =\left[1-[\bar{X}-1]^{2}\right][\bar{X}-1]^{-2} i \not \partial-V \frac{1}{m}[\bar{X}-1]^{-2} i \not \partial \\
& =\left(1-V \frac{1}{m}\right)[\bar{X}-1]^{-2} i \not \partial-i \not \partial \\
& =\left(1-V \frac{1}{m}\right)[\bar{X}-1]^{-2}(i \not \partial+m)-\left(1-V \frac{1}{m}\right)[\bar{X}-1]^{-2} m-i \not \partial \\
& =\left(1-V \frac{1}{m}\right)[\bar{X}-1]^{-2}(i \not \partial+m)-i \not \partial-\left(1-V \frac{1}{m}\right)\left([\bar{X}-1]^{-2}(i \not \partial+m) \frac{1}{m} V+m\right) \\
& =\left(1-V \frac{1}{m}\right)[\bar{X}-1]^{-1}(i \not \partial+m)[X-1]^{-1}\left(1-\frac{1}{m} V\right)-i \not \partial-m+V .
\end{aligned}
$$

Here, in the second, fifth and sixth lines we used Eqs. (3.5), (A.2) and (A.3), respectively. Inserting Eq. A.1 into the last line of the above equation we complete the proof of Eq. (A.4).

At the end of this appendix we would like to prove an additional relation used in the first line of Eq. (3.9)

$$
i \not \partial-m+V=2 \bar{S} \bar{X}^{-1}(i \not \partial+m)-2 m S
$$

To derive this relation we use the commutation-like relation

$$
(i \not \partial+m) X^{-1}=\bar{X}^{-1}(i \not \partial+m)
$$

as well as the "conjugate" of Eq. (3.14)

$$
\frac{1}{m} V \frac{1}{m}(i \not \partial+m) X^{-1}=2-X
$$


With these relations the derivation of the first line becomes obvious:

$$
\begin{aligned}
i \not \partial-m+V & =i \not \partial+m-2 m+V=i \not \partial+m-m(2-X)-2 m S \\
& =i \not \partial+m-V \frac{1}{m}(i \not \partial+m) X^{-1}-2 m S=i \not \partial+m-V \frac{1}{m} \bar{X}^{-1}(i \not \partial+m)-2 m S \\
& =\left(1-V \frac{1}{m} \bar{X}^{-1}\right)(i \not \partial+m)-2 m S=2 \bar{S} \bar{X}^{-1}(i \not \partial+m)-2 m S .
\end{aligned}
$$

[1] K. Johnson and E. C. G. Sudarshan, Annals Phys. 13, 126 (1961).

[2] G. Velo and D. Zwanziger, Phys. Rev. 186, 1337 (1969).

[3] M. Benmerrouche, R. M. Davidson and N. C. Mukhopadhyay, Phys. Rev. C 39 (1989) 2339.

[4] V. Pascalutsa, M. Vanderhaeghen and S. N. Yang, Phys. Rept. 437 (2007) 125 arXiv:hep-ph/0609004.

[5] V. Bernard, Prog. Part. Nucl. Phys. 60 (2008) 82 arXiv:0706.0312 [hep-ph]].

[6] P.A. Moldauer, K.M. Case, Phys. Rev. 102, 279 (1955).

[7] V. Pascalutsa, Phys. Rev. D 58, 096002 (1998) arXiv:hep-ph/9802288.

[8] S. R. Coleman, J. Wess and B. Zumino, Phys. Rev. 177 (1969) 2239.

[9] C. G. Callan, S. R. Coleman, J. Wess and B. Zumino, Phys. Rev. 177 (1969) 2247.

[10] V. Pascalutsa, Phys. Lett. B 503, 85 (2001) arXiv:hep-ph/0008026].

[11] P. A. M. Dirac, Lectures on Quantum Mechanics, Dover Publications, 2001.

[12] L. D. Faddeev, Theor. Math. Phys. 1 (1970), 1.

[13] G. Senjanovic, Phys. Rev. D 16 (1977) 307.

[14] N. Wies, J. Gegelia and S. Scherer, Phys. Rev. D 73 (2006) 094012 arXiv:hep-ph/0602073.

[15] R. Haag, Phys. Rev. 112, 669 (1958).

[16] H. B. Tang and P. J. Ellis, Phys. Lett. B 387 (1996) 9 arXiv:hep-ph/9606432.

[17] P. J. Ellis and H. B. Tang, Phys. Rev. C 56, 3363 (1997) arXiv:hep-ph/9609459.

[18] N. Fettes and U.-G. Meißner, Nucl. Phys. A 679 (2001) 629 arXiv:hep-ph/0006299.

[19] T. R. Hemmert, B. R. Holstein and J. Kambor, J. Phys. G 24 (1998) 1831 arXiv:hep-ph/9712496]. 